Comment. Math. Helv. 75 (2000) 247-254

(C) 2000 Birkhäuser Verlag, Basel

\title{
Compact minimal hypersurfaces with index one in the real projective space
}

Manfredo do Carmo, Manuel Ritoré and Antonio Ros

Abstract. Let $M^{n}$ be a compact (two-sided) minimal hypersurface in a Riemannian manifold $\bar{M}^{n+1}$. It is a simple fact that if $\bar{M}$ has positive Ricci curvature then $M$ cannot be stable (i. e. its Jacobi operator $L$ has index at least one). If $\bar{M}=S^{n+1}$ is the unit sphere and $L$ has index one, then it is known that $M$ must be a totally geodesic equator.

We prove that if $\bar{M}$ is the real projective space $P^{n+1}=S^{n+1} /\{ \pm\}$, obtained as a metric quotient of the unit sphere, and the Jacobi operator of $M$ has index one, then $M$ is either a totally geodesic sphere or the quotient to the projective space of the hypersurface $S^{n_{1}}\left(R_{1}\right) \times S^{n_{2}}\left(R_{2}\right) \subset$ $S^{n+1}$ obtained as the product of two spheres of dimensions $n_{1}, n_{2}$ and radius $R_{1}, R_{2}$, with $n_{1}+n_{2}=n, R_{1}^{2}+R_{2}^{2}=1$ and $n_{1} R_{2}^{2}=n_{2} R_{1}^{2}$.

Mathematics Subject Classification (2000). Primary 53A10; Secondary 53A05, 53C42.

\section{Introduction}

Given a compact minimal hypersurface (without boundary) $M$ in a compact Riemannian manifold $\bar{M}^{n+1}$, the second variation formula for the area determines on the normal bundle of $M$ a selfadjoint elliptic operator $L$ called the Jacobi operator of $M$. If the normal bundle of $M$ is trivial or, in other words, if $M$ has a globally defined unit normal vector field $N$, then we say that $M$ is twosided. When $\bar{M}$ is orientable, this property is equivalent to the orientability of $M$. In the two-sided case the Jacobi operator acts on functions and it is given by $L u=\Delta u+\left(\overline{\operatorname{Ric}}(N)+|\sigma|^{2}\right) u$, for any $u \in C^{\infty}(M)$, where $\Delta$ is the Laplacian of $M$, $\overline{\operatorname{Ric}}(N)$ is the Ricci curvature of $\bar{M}$ in the direction of the normal vector $N$ and $|\sigma|$ is the length of the second fundamental form of the immersion. The index of $M$ is defined as the number of negative eigenvalues of $L$. If the index is zero, then $M$ is said to be stable. Although stability play an important role in the theory of minimal hypersurfaces it is easy to see that some manifolds $\bar{M}$ admit no two-sided stable compact hypersurfaces: this holds, for instance, if the Ricci curvature of $\bar{M}$

First author supported by CNPq and PRONEX. Second and third authors partially supported by DGICYT research group PB97-0785. 
is positive. On the other hand, Pitts [11] has proved that any compact Riemannian manifold $\bar{M}$ admits an embedded compact minimal hypersurface with index less than or equal to one (which is free of singularities when $n \leqslant 7$ ). Also Pitts and Rubinstein [12] have produced, by minimax method a certain number of index one examples in three-manifolds. Ross [16] has proved that the classical Schwarz surface has index one in the cubic flat three torus.

If the ambient manifold has nonnegative Ricci curvature it is natural to hope that the family of two-sided compact index one minimal hypersurfaces has specific nice properties. In particular complete classifications of these hypersurfaces seems to be possible for ambient spaces $\bar{M}$ simple enough. The constant curvature case, i. e., when $\bar{M}$ is a flat or elliptic space form, is of special interest. At the present there are few classifying results, even for simple three manifolds. We have the following results: Simons [17] proved that index one minimal surfaces in the sphere are totally geodesic. López and Ros [9], using results by Fischer-Colbrie [7], showed that the only complete minimal surfaces with index one are the Catenoid and Enneper surface. Previously do Carmo and Peng [6] and Fischer-Colbrie and Schoen [8] had shown that the only stable (index zero) complete minimal surface is the plane. From the work by Ritore and Ros [14] a classification of index one minimal surfaces in $\mathbb{P}^{3}$ can be obtained: it must be a two-fold covering of a linear subvariety or a tube of certain radius around a line. These authors [15] also obtained a compactness result for the space of index one minimal surfaces in flat three tori. They prove that the set of flat three tori that admit embedded orientable compact minimal surfaces with index one is a compact subset in the moduli space. Ritoré ([13]) made a study of index one minimal surfaces in flat three space forms. For general 3-dimensional ambient space some partial results are known. The interested reader can consult [14] and the references there.

In this paper we treat compact two-sided index one minimal hypersurfaces in the real projective space $\mathbb{P}^{n+1}$. Our main result, Theorem 3 , is

The only compact two-sided minimal hypersurfaces with index one in the real projective space $\mathbb{P}^{n+1}$ are the totally geodesic spheres and the minimal Clifford hypersurfaces.

The first ones are the twofold covering of the linear hypersurfaces (which are one-sided) while the Clifford hypersurfaces are embedded. These hypersurfaces are simply the quotient to $\mathbb{P}^{n+1}$ of the product of two spheres of right dimension and radii that lie in $\mathbb{S}^{n+1}$. They are defined in section 1 .

Recall that a constant mean curvature hypersurface in an $(n+1)$-dimensional manifold is volume preserving stable if the second derivative of the $n$-volume is nonnegative for variations preserving the $(n+1)$-volume enclosed ([1]). An important remark is that the boundary of the isoperimetric domains of $\bar{M}$ are volume preserving stable. Using this fact Ritoré and Ros give in [14] a complete solution of the isoperimetric problem in the three dimensional projective space. As a consequence of Theorem 3 we obtain in Theorem 4 a classification of the volume preserving stable two-sided hypersurfaces in $\mathbb{P}^{n+1}$ which are minimal. 
The only compact two-sided minimal hypersurfaces which are volume preserving stable in the real projective space $\mathbb{P}^{n+1}$ are the totally geodesic spheres and the minimal Clifford hypersurfaces.

We have organized this paper into two sections. In the first one we define and study Clifford hypersurfaces. In the second one we state and prove our results.

The first author wishes to thank the warm hospitality of the Department of Geometry and Topology of the University of Granada where this paper was written.

\section{Clifford hypersurfaces}

In this section we review the basic properties of a simple family of hypersurfaces in the unit sphere $\mathbb{S}^{n+1} \subset \mathbb{R}^{n+2}$. Given two positive integers $n_{1}$ and $n_{2}$ with $n_{1}+n_{2}=n$ and two positive real numbers $R_{1}$ and $R_{2}$ such that $R_{1}^{2}+R_{2}^{2}=1$, the product $\mathbb{S}^{n_{1}}\left(R_{1}\right) \times \mathbb{S}^{n_{2}}\left(R_{2}\right)$ of the spheres $\mathbb{S}^{n_{i}}\left(R_{i}\right)=\left\{p_{i} \in \mathbb{R}^{n_{i}+1}:\left|p_{i}\right|=R_{i}\right\}$, $i=1,2$, is a compact homogeneous hypersurface of the sphere $\mathbb{S}^{n+1}$ usually called a Clifford hypersurface.

If $p=\left(p_{1}, p_{2}\right)$ is a point in $M=\mathbb{S}^{n_{1}}\left(R_{1}\right) \times \mathbb{S}^{n_{2}}\left(R_{2}\right)$, then a unit vector normal to $M$ at this point is given by

$$
N=\left(-\frac{R_{2}}{R_{1}} p_{1}, \frac{R_{1}}{R_{2}} p_{2}\right) .
$$

Therefore the principal curvatures of $M$ are $\frac{R_{2}}{R_{1}}$, with multiplicity $n_{1}$, and $-\frac{R_{1}}{R_{2}}$, which has multiplicity $n_{2}$. In particular,

$$
M \text { minimal } \Longleftrightarrow n_{1} R_{2}^{2}=n_{2} R_{1}^{2} \text {. }
$$

Now we assume that $M$ is a minimal Clifford hypersurface. Note that there is just one of such hypersurfaces for any choice of $n_{1}$ and $n_{2}$. The square length of the second fundamental form of $M$ is given by

$$
|\sigma|^{2}=n_{1} \frac{R_{2}^{2}}{R_{1}^{2}}+n_{2} \frac{R_{1}^{2}}{R_{2}^{2}}=n .
$$

As the Ricci curvature of $\mathbb{S}^{n+1}$ is equal to $n$, it follows that the Jacobi operator of $M$ is simply $L=\Delta+2 n$. The eigenvalues of the Laplacian of $M$ are known to be

$$
\frac{k_{1}\left(k_{1}+n_{1}-1\right)}{R_{1}^{2}}+\frac{k_{2}\left(k_{2}+n_{2}-1\right)}{R_{2}^{2}}
$$

where $k_{1}$ and $k_{2}$ are nonnegative integers, see [3]. 
The hypersurface $M$ is invariant under the antipodal map and, so, it induces an embedded minimal hypersurface $M /\{ \pm\}$ in the real projective space $\mathbb{P}^{n+1}=$ $\mathbb{S}^{n+1} /\{ \pm\}$, which we will also call a Clifford hypersurface. The Jacobi operator of $M /\{ \pm\}$ is given again by $L=\Delta+2 n$, but the eigenvalues of the Laplacian in the quotient hypersurface are only those in (1) for which $k_{1}+k_{2}$ is even. In particular, the first non zero eigenvalue of the Laplacian of $M /\{ \pm\}$ corresponds to $k_{1}=k_{2}=1$ (the other candidates are obtained for $\left(k_{1}, k_{2}\right)=(2,0),(0,2)$, but it can be checked directly that they give bigger eigenvalues) and its value is

$$
\frac{n_{1}}{R_{1}^{2}}+\frac{n_{2}}{R_{2}^{2}}=n_{1} \frac{R_{1}^{2}+R_{2}^{2}}{R_{1}^{2}}+n_{2} \frac{R_{1}^{2}+R_{2}^{2}}{R_{2}^{2}}=n_{1}+n_{2}+n_{1} \frac{R_{2}^{2}}{R_{1}^{2}}+n_{2} \frac{R_{1}^{2}}{R_{2}^{2}}=2 n
$$

Therefore it follows that for any minimal Clifford hypersurface $M$ in $\mathbb{S}^{n+1}$, the induced hypersurface in the projective space, $M /\{ \pm\}$, has index one.

\section{Results}

Let $f: M \rightarrow \mathbb{S}^{n+1}$ be an orientable compact minimal hypersurface of the sphere and $N$ its unit normal vector field. These maps verify the differential equations

$$
\Delta f+n f=0 \quad \text { and } \quad \Delta N+|\sigma|^{2} N=0
$$

where $|\sigma|$ is the length of the second fundamental form of the immersion. The Jacobi operator of $M$ is given by $L=\Delta+|\sigma|^{2}+n$ and its associated quadratic form is

$$
Q(u, u)=-\int_{M} u L u d V=\int_{M}\left\{|\nabla u|^{2}-\left(|\sigma|^{2}+n\right) u^{2}\right\} d V,
$$

for any smooth function $u$ on $M$.

Given $\mathbf{a}, \mathbf{b} \in \mathbb{R}^{n+2}$ we consider the vector valued function $\phi_{\mathbf{a}, \mathbf{b}}: M \rightarrow \mathbb{R}^{n+2}$ defined by

$$
\phi_{\mathbf{a}, \mathbf{b}}=\langle f, \mathbf{a}\rangle f+\langle N, \mathbf{a}\rangle N+\langle f, \mathbf{b}\rangle N .
$$

Lemma 1. The value of the Jacobi operator when applied to the function $\phi_{\mathbf{a}, \mathbf{b}}$ is given by

$$
-L \phi_{\mathbf{a}, \mathbf{b}}=\left(n-|\sigma|^{2}\right)(\langle f, \mathbf{a}\rangle f-\langle N, \mathbf{a}\rangle N)+X,
$$

where $X: M \rightarrow \mathbb{R}^{n+2}$ is a vector field tangent to $M$.

Proof. Each one of the summands of $\phi \mathbf{a}, \mathbf{b}$ is a product of two functions, say $u$ and $v$. The lemma follows by combining the formula $\Delta(u v)=v \Delta u+u \Delta v+2\langle\nabla u, \nabla v\rangle$ with equations (2) and using the fact that, in our case, the terms which correspond to the product of gradients are always tangent to $M$. 
Lemma 2. Given $\mathbf{a}, \mathbf{b} \in \mathbb{R}^{n+2}$ we have

$$
\int_{M}\left(|\sigma|^{2}-n\right)\langle N, \mathbf{a}\rangle\langle f, \mathbf{b}\rangle d V=0 .
$$

Proof. Using (2) and Green's theorem we get

$$
\int_{M}\left(|\sigma|^{2}-n\right)\langle N, \mathbf{a}\rangle\langle f, \mathbf{b}\rangle d V=\int_{M}(\langle N, \mathbf{a}\rangle \Delta\langle f, \mathbf{b}\rangle-\langle f, \mathbf{b}\rangle \Delta\langle N, \mathbf{a}\rangle) d V=0,
$$

as we claimed.

As remarked in the introduction, there are no stable two-sided hypersurfaces in $\mathbb{P}^{n+1}$ since its Ricci curvature is strictly positive. Examples of compact twosided hypersurfaces with index one in $\mathbb{P}^{n+1}$ are the totally geodesic immersions of $\mathbb{S}^{n}$ in $\mathbb{P}^{n+1}$ (twofold coverings of embedded totally geodesic $\mathbb{P}^{n}$ ) and the Clifford hypersurfaces in $\mathbb{P}^{n+1}$. Let us see that they the only examples.

Theorem 3. The only compact two-sided minimal hypersurfaces with index one in the real projective space $\mathbb{P}^{n+1}$ are the totally geodesic spheres and the minimal Clifford hypersurfaces.

Moreover the only embedded ones are the Clifford hypersurfaces.

Proof. Let $\widetilde{f}: \widetilde{M} \rightarrow \mathbb{P}^{n+1}$ be a two-sided index one compact minimal hypersurface. By using locally constant test functions we conclude from the index one assumption that $\widetilde{M}$ must be connected. If $\widetilde{f}$ lifts to an immersion of $\widetilde{M}$ to the sphere $\mathbb{S}^{n+1}$, then $\widetilde{M}$ is an orientable index one minimal hypersurface of the sphere. It follows from [17] that, in this case, $\widetilde{M}$ is a totally geodesic sphere.

Henceforth we assume that the above lift does not exist. Therefore there is a connected twofold covering $M \rightarrow \widetilde{M}$ and a isometric minimal immersion $f$ : $M \rightarrow \mathbb{S}^{n+1}$ locally congruent to $\tilde{f}$ and such that, if we denote by $s: M \rightarrow M$ the isometric involution induced by the covering, then $f$ is odd, that is $f \circ s=-f$. Moreover, the two-sidedness of $\widetilde{M}$ implies that $M$ is orientable and that its unit normal vector field $N: M \rightarrow \mathbb{S}^{n+1}$ also verifies $N \circ s=-N$. In particular the functions $\phi_{\mathbf{a}, \mathbf{b}}$ above are even with respect to $s$, i. e., $\phi_{\mathbf{a}, \mathbf{b}} \circ s=\phi_{\mathbf{a}, \mathbf{b}}$. Observe that the first eigenfunction $\varphi$ of the Jacobi operator $L$ of $M$ is also even: this follows because the associated eigenspace is one dimensional, $s$ is an isometry and $\varphi>0$ on $M$.

Our index one hypothesis, when translated to $M$, says that $Q(u, u) \geqslant 0$ for any smooth function $u$ on $M$ such that $u \circ s=u$ and $\int_{M} u \varphi d V=0$. Moreover, if for a function $u$ as above we have $Q(u, u)=0$, then $u$ is a Jacobi function, that is $L u=0$ on $M$.

In our argument we will use as test functions the maps $\phi_{\mathbf{a}, \mathbf{b}}$ which are even and that, under suitable choice of the parameters $\mathbf{a}, \mathbf{b}$ in $\mathbb{R}^{n+2}$ will be orthogonal 
to $\varphi$. From Lemmae 1 and 2 we obtain

$$
Q\left(\phi_{\mathbf{a}, \mathbf{b}}, \phi_{\mathbf{a}, \mathbf{b}}\right)=\int_{M}\left(n-|\sigma|^{2}\right)\left(\langle f, \mathbf{a}\rangle^{2}-\langle N, \mathbf{a}\rangle^{2}\right) d V .
$$

Note that the expression above does not depend on $\mathbf{b}$. Consider the linear map $F: \mathbb{R}^{n+2} \rightarrow \mathbb{R}^{n+2}$ defined, for any $\mathbf{b} \in \mathbb{R}^{n+2}$, by

$$
F(\mathbf{b})=\int_{M} \varphi\langle f, \mathbf{b}\rangle N d V .
$$

Claim. $F$ is a linear isomorphism.

Proof. To prove this claim assume, reasoning by contradiction, that there is $\mathbf{b} \neq$ 0 such that $F(\mathbf{b})=0$. Taking $\phi=\phi_{\mathbf{0}, \mathbf{b}}=\langle f, \mathbf{b}\rangle N$, we have from (4) that $Q(\phi, \phi)=0$. Thus $L \phi=0$. On the other hand, Lemma 1 says that $L \phi$ is a certain tangent vector field $X$ along $M$. Explicit computation gives, in this special case, that $X=-A \mathbf{b}^{t}$, where $A$ is the second fundamental form of $M$, viewed as an endomorphism, and $\mathbf{b}^{t}$ is the tangent part of $\mathbf{b}$ along $M$. Thus we have that $A \mathbf{b}^{t}=0$ on $M$, which is the same to say that the function $\langle N, \mathbf{b}\rangle$ is constant. As $N$ is an odd function, this constant must be zero. From that we see that the Hessian of the linear function $u=\langle f, \mathbf{b}\rangle$ is given by $-\langle\rangle$,$u . If u \neq \equiv 0$, then Obata's theorem ([3]) asserts that $M$ is isometric to a unit sphere. In this case the Gauss equation implies that $M$ is totally geodesic in $\mathbb{S}^{n+1}$. Thus $M$ is either a linear hypersurface in the projective space (which cannot hold because these hypersurfaces are one-sided) or a totally geodesic sphere covering twice a linear hypersurface (which is again not possible because this immersion lifts to the $(n+1)$-dimensional sphere). If $u \equiv 0$, then we conclude, now in a trivial way, that $M$ is again totally geodesic which is impossible as above. This contradiction proves the claim.

Take an orthonormal basis $\mathbf{a}_{1}, \ldots, \mathbf{a}_{n+2}$ in $\mathbb{R}^{n+2}$. For any $i=1, \ldots, n+2$ we can find, using the claim above, a vector $\mathbf{b}_{i} \in \mathbb{R}^{n+2}$ such that the function $\phi_{i}=\phi_{\mathbf{a}_{i}, \mathbf{b}_{i}}$ is $L^{2}$-orthogonal to $\varphi$. Therefore $Q\left(\phi_{i}, \phi_{i}\right) \geqslant 0$ and from (4) we get

$0 \leqslant \sum_{i=1}^{n+2} \int_{M}\left(n-|\sigma|^{2}\right)\left\{\left\langle f, \mathbf{a}_{i}\right\rangle^{2}-\left\langle N, \mathbf{a}_{i}\right\rangle^{2}\right\} d V=\int_{M}\left(n-|\sigma|^{2}\right)\left(|f|^{2}-|N|^{2}\right) d V=0$.

This implies that $L \phi_{i}=0$ for $i=1, \ldots, n+2$ and so, using lemma 1 we conclude that $\left(n-|\sigma|^{2}\right)\left\langle f, \mathbf{a}_{i}\right\rangle=0$ for any $i$, which is possible only if $n-|\sigma|^{2}=0$ on $M$. Now the result of Chern, do Carmo and Kobayashi [5] says that $M$ is locally congruent to a Clifford minimal hypersurface. Thus $M$ is congruent either to the Clifford hypersurface $\mathbb{S}^{n_{1}}\left(R_{1}\right) \times \mathbb{S}^{n_{2}}\left(R_{2}\right) \subset \mathbb{S}^{n+1}$ (with $n_{1} R_{2}^{2}=n_{2} R_{1}^{2}$ ) itself or to 
a nontrivial finite covering of it. We discard the second case (which, of course, is possible only if either $n_{1}$ or $n_{2}$ are equal to one) because its index is bigger than one. We can see that by checking explicitly the eigenvalues of the Laplacian, as in (1). This proves the theorem.

A compact constant mean curvature hypersurface $M^{n}$ immersed in $\bar{M}^{n+1}$ is volume preserving stable if the second derivative of the $n$-volume is nonnegative for any variation keeping constant the $(n+1)$-volume. If $\bar{M}=\mathbb{P}^{n+1}$ and $M$ is two-sided then volume preserving stability is equivalent to

$$
-\int_{M} u\left(\Delta u+\left(|\sigma|^{2}+n\right) u\right) d V \geqslant 0
$$

for any smooth function $u$ on $M$ with mean zero, where $|\sigma|$ is the square of the norm of the second fundamental form $\sigma$ of $M$. A complete classification of compact twosided volume preserving stable surfaces in $\mathbb{P}^{3}$ is given in [14]. As a consequence, the isoperimetric domains in $\mathbb{P}^{3}$ are found.

If $M$ is volume preserving stable then the operator $\Delta+|\sigma|^{2}+n$ has index zero or one (none or one negative eigenvalues). As $|\sigma|^{2}+n>0$ then it cannot have index zero. So a compact minimal hypersurface which is two-sided and volume preserving stable has index one. By Theorem 3, $M$ must be a totally geodesic sphere or a Clifford hypersurface. Since $|\sigma|^{2}+n$ is constant for these hypersurfaces, checking volume preserving stability is reduced to an eigenvalue comparison as in section 1 $([1])$. So we have

Theorem 4. The only compact two-sided minimal hypersurfaces which are volume preserving stable in the real projective space $\mathbb{P}^{n+1}$ are the totally geodesic spheres and the minimal Clifford hypersurfaces.

It is expected that, as in the three dimensional case, the isoperimetric domains in $\mathbb{P}^{n+1}$ are some geodesic balls and their complementary domains, and the domains enclosed by some Clifford hypersurfaces, see Berger [2, pp. 141-142] and Burago and Zalgaller [4, 10.2.3].

The main results in this paper remain valid if we allow a singular set of zero $s$-dimensional Hausdorff measure, with $s \geqslant n-2$ (for instance for solutions to the isoperimetric problem), see [10].

\section{References}

[1] J. L. Barbosa, M. do Carmo and J. Eschenburg, Stability of hypersurfaces with constant mean curvature in Riemannian manifolds, Math. Z., 197 (1988), 123-138.

[2] M. Berger, Riemannian geometry during the second half of the twentieth century, Jber. d. Dt. Math.-Verein. 100 (1998) 45-208. 
[3] M. Berger, Gauduchon and Mazet, Le spectre d'une variété Riemanniene, Lecture Notes in Math. 64, Springer-Verlag.

[4] Y. D. Burago, V. A. Zalgaller, Geometric Inequalities, Grund. Math. Wissen. 285, Springer-Verlag, Berlín, 1988.

[5] S. S. Chern, M. do Carmo and S. Kobayashi, Minimal submanifolds of a sphere with second fundamental form of constant length, Functional Analysis and Related Fields, Springer 1970, 59-75.

[6] M. do Carmo and C. K. Peng, Stable complete minimal surfaces in $\mathbb{R}^{3}$ are planes, Bull. Amer. Math. Soc. (N.S.), 1 (1979), 903-906.

[7] D. Fisher-Colbrie, On complete minimal surfaces with finite Morse index in three-manifolds, Invent. Math. 82 (1985), 121-132.

[8] D. Fischer-Colbrie and R. Schoen, The structure of complete stable minimal surfaces in 3-manifolds of non-negative scalar curvature, Comm. Pure Appl. Math., 33 (1980), 199211.

[9] F. J. López and A. Ros, Complete minimal surfaces with index one and stable constant mean curvature surfaces, Comm. Math. Helv. 64 (1989), 34-43.

[10] F. Morgan and M. Ritoré, Isoperimetric regions in cones, preprint, 2000.

[11] J. T. Pitts, Existence and regularity of minimal surfaces on Riemannian manifolds, Princeton University Press, New Jersey, 1981.

[12] J. T. Pitts and J. H. Rubinstein, Existence of minimal surfaces of bounded topological type in three manifolds Proc. Centre for Mathematical Analysis, Ed. L. Simon and N. Trudinger 10 (1985), 163-176.

[13] M. Ritoré, Index one minimal surfaces in flat three space forms, Indiana Univ. Math. J. 46 (1997), 1137-1153.

[14] M. Ritoré and A. Ros, Stable constant mean curvature tori and the isoperimetric problem in three space forms, Comm. Math. Helv. 67 (1992), 293-305.

[15] M. Ritoré and A. Ros, The spaces of index one minimal surfaces and stable constant mean curvature surfaces in flat three manifolds, Trans. Amer. Math. Soc. 348 (1996), 391-410.

[16] M. Ross, Schwarz's $\mathcal{P}$ and $\mathcal{D}$ surfaces are stable, Diff. Geom. and Appl. 2 (1992), 179-195.

[17] J. Simons, Minimal varieties in Riemannian manifolds, Ann. of Math. 88 (1968), 62-105.

Manfredo do Carmo

Instituto de Matematica Pura e Aplicada

Estrada Dona Castorina

Rio de Janeiro

Brasil

e-mail: manfredo@impa.br

Manuel Ritoré and Antonio Ros

Departamento de Geometría y Topología

Universidad de Granada

E-18071 Granada

España

e-mail: ritore@ugr.es

aros@ugr.es

(Received: June 6, 1998) 